\title{
Efficient Transmission Electron Microscopy Characterization of Cell- Nanostructure Interfacial Interactions
}

Stella Aslanoglou ${ }^{\dagger}$, Yaping Chen ${ }^{\dagger}$, Viola Oorschot, Zlatan Trifunovic, Eric Hanssen, Koukou Suu, Nicolas H. Voelcker* and Roey Elnathan*

\section{Experimental section}

\section{Substrate cleaning:}

Flat silicon wafers ( $3^{\prime \prime} / 4 "$, p-type, $3-6 \Omega \mathrm{cm},<100>$, Siltronix, France) were cleaned by sonication in 1:1 solution of ethanol:acetone for $5 \mathrm{~min}$ and then sonicated again in MilliQ water for $5 \mathrm{~min}$. This was followed by dipping the wafers into boiling Piranha solution $\left(3: 1 \mathrm{H}_{2} \mathrm{SO}_{4}: \mathrm{H}_{2} \mathrm{O}_{2} \mathrm{v} / \mathrm{v}, 75{ }^{\circ} \mathrm{C}\right.$, Avantor Performance Materials) for $1 \mathrm{~h}$ to remove any organic contaminants, then washing with water and drying under a nitrogen gas jet.

\section{SiNW arrays fabrication:}

Convective Assembly Deposition: Hexagonally close-packed polystyrene microspheres (PSMS) monolayers were deposited over quarter pieces of a 3" Si wafer by convective assembly. The apparatus included a mounted microscope slide that was used as a blade for the PSMS depositions, a $50 \mathrm{~mm}$ motorized translation stage (MTS50-Z8, Thorlabs, Inc.), and a compact sub-Hertz pendulum vibration isolation system (TMC). The blade was adjusted to leave a small space between the bottom edge of the blade and the Si substrate. $25 \mu \mathrm{L}$ of a suspension of PSMS (polybead microspheres solutions, $3 \mu \mathrm{m}$ in diameter, $2.5 \% \mathrm{w} / \mathrm{v}$ in water) was injected into the space between the blade and the sample using a micropipette, forming a meniscus between the pinned substrate and the bottom edge of the blade. This resulted in a continuous contact line of PSMS suspension on the Si substrate. To deposit PSMS in a uniform monolayer, operating parameters such as stage velocity and PS suspension concentration were adjusted.

Oxygen Plasma Reactive Ion Etching (RIE) of Polystyrene Microspheres: Samples prepared by convective assembly were inserted into Plasmalab100 ICP380 deep reactive ion etcher (Oxford Instruments), where oxygen plasma treatment was performed in order to reduce the size of the PSMS. During the etching step, a flow rate of $100 \mathrm{sccm} \mathrm{O}_{2}$ was used with inductively coupled plasma (ICP) power of $100 \mathrm{~W}$ and bias power of $50 \mathrm{~W}$. The APC valve position was set at 35 and He pressure was set at 10 Torr. The reduced size PSMS served as a mask for the subsequent silicon etching in two steps. 
Deep RIE of Silicon: 1) Bosch process: Silicon etching was performed by alternate cycles of passivation and etching steps to obtain SiNWs with a cylindrical profile. During the passivation step (6 s), a flow rate of $150 \mathrm{sccm} \mathrm{C}_{4} \mathrm{~F}_{8}$ and $3 \mathrm{sccm} \mathrm{SF}_{6}$ was used with ICP power of $1500 \mathrm{~W}$ and bias power of $5 \mathrm{~W}$. During the etching step $(8 \mathrm{~s})$, a flow rate of $150 \mathrm{sccm} \mathrm{SF} 6$ and $3 \mathrm{sccm} \mathrm{C}_{4} \mathrm{~F}_{8}$ was used with an ICP power of $2000 \mathrm{~W}$ and a bias power of $20 \mathrm{~W}$. The APC valve position was set at 70 and Helium (He) pressure at 10 Torr in both steps. The etching depth of Si was controlled by the number of cycles. Following the Bosch process, PSMS were removed by sonication of the samples in MilliQ water. Samples were then dried under a nitrogen gas jet and inserted back to the deep reactive ion etcher.

2) Pseudo-Bosch process: Silicon etching was performed in a simultaneous flow of $100 \mathrm{sccm} \mathrm{SF}_{6}$ and $40 \mathrm{sccm} \mathrm{C}_{4} \mathrm{~F}_{8}$ at a pressure of $10 \mathrm{mTorr}$, with ICP power of $1500 \mathrm{~W}$ and bias power of $50 \mathrm{~W}$ to achieve a conically shaped profile of the SiNWs. He pressure was set at 10 Torr. The etching time was $115 \mathrm{~s}$.

\section{SiNT arrays fabrication:}

E-beam Lithography: HSQ resist (XR-1541-002, Dow Corning, USA) was spin coated onto a silicon wafer with a spin speed of $1500 \mathrm{rpm}$ for $1 \mathrm{~min}$. The sample was directly loaded into an electron beam lithography (EBL) system (VISTEC EBPG-5000+, Raith Company, Germany) without baking. The EBL was performed at an accelerating voltage of $100 \mathrm{kV}$ with a beam current of $30 \mathrm{nA}$, using a dose of $1000 \mu \mathrm{Ccm}^{-2}$. After e-beam exposure, the HSQ resist was developed using AZ 726 MIF. Development was stopped with water and samples were dried under a nitrogen gas jet.

Deep Reactive Ion Etching (DRIE) of Silicon: Samples prepared by e-beam lithography were inserted into an ULVAC NLD5700 DRIE. Silicon etching was performed in a simultaneous flow of SF 6 and $\mathrm{O}_{2}$ at a pressure of $1 \mathrm{~Pa}$ with Antenna RF power of $200 \mathrm{~W}$ and Bias RF LF power of $16 \mathrm{~W}$. He pressure was set at $2000 \mathrm{~Pa}$ and the circulator at $-20{ }^{\circ} \mathrm{C}$. The etching time was $145 \mathrm{~s}$.

\section{Cell culture:}

GPE86 cells (ATCC, CRL-9642) and HeLa cells (ATCC, CCL-2) were grown and maintained in Dulbecco's modified Eagle's medium (DMEM, Gibco) supplemented with 10\% fetal bovine serum (FBS, Gibco), $1 \mathrm{mM}$ sodium pyruvate (Gibco), $2 \mathrm{mM}$ L-glutamine (Gibco), $100 \mathrm{U} \mathrm{mL}^{-1}$ penicillin (Gibco), and $100 \mu \mathrm{g} \mathrm{mL}^{-1}$ streptomycin (Gibco). Cells were incubated at $37{ }^{\circ} \mathrm{C}$ with $5 \% \mathrm{CO}_{2}$.

\section{SEM sample preparation:}

Cells grown on SiNW/SiNT substrates were rinsed with $0.1 \mathrm{M}$ sodium cacodylate buffer (Electron Microscopy Sciences) and fixed with 2.5\% glutaraldehyde (Electron Microscopy Sciences) in $0.1 \mathrm{M}$ 
sodium cacodylate at $4{ }^{\circ} \mathrm{C}$ overnight. Following this, substrates were washed $(3 \times 5 \mathrm{~min})$ with chilled $0.1 \mathrm{M}$ sodium cacodylate buffer and post-fixed with 1\% osmium tetroxide (Electron Microscopy Sciences) in $0.1 \mathrm{M}$ sodium cacodylate at RT for $1 \mathrm{~h}$. After repeating the washing step, substrates were gradually dehydrated with increasing concentrations of ethanol; 50\%, 70\%, 90\% $(1 \times 10 \mathrm{~min})$ and $100 \%(2 \times 10 \mathrm{~min})$ at RT, and finally were critical point dried (CPD 030 Critical Point Dryer, BALTEC). Substrates were then mounted on SEM stubs and sputter coated with a $7 \mathrm{~nm}$ layer of either gold or platinum in order to increase their conductivity.

\section{SEM Imaging:}

SEM imaging of resin imprints and SiNW/SiNT substrates with cells was performed using a Thermo Fisher Nova NanoSEM 430. The images were taken at tilt $\left(45^{\circ}\right)$ or top views, and at magnifications of $500 \mathrm{X}, 2 \mathrm{kX}, 7 \mathrm{kX}$, and $10 \mathrm{kX}$, with an electron beam acceleration voltage of $3-5 \mathrm{kV}$ and a current of $80 \mathrm{pA}$, while using a secondary electron detector.

\section{FIB-SEM sample preparation:}

The sample preparation combined heavy metal staining with resin embedding. In particular, samples were rinsed with $0.1 \mathrm{M}$ sodium cacodylate buffer and fixed with $2.5 \%$ glutaraldehyde in the same buffer at $4{ }^{\circ} \mathrm{C}$ overnight. Following this, samples were washed $(3 \times 5 \mathrm{~min})$ with chilled $0.1 \mathrm{M}$ sodium cacodylate buffer and quenched with chilled $20 \mathrm{mM}$ glycine solution (Sigma-Aldrich) in the same buffer for $20 \mathrm{~min}$. After repeating the washing step, samples were post-fixed by combining equal volumes of $4 \%$ aqueous osmium tetroxide with $2 \%$ potassium ferrocyanide (UNIVAR) in $0.2 \mathrm{M}$ sodium cacodylate buffer on ice for $1 \mathrm{~h}$. Samples were then washed again $(3 \times 5 \mathrm{~min})$ with chilled buffer and incubated with 1\% tannic acid (BDH) in water at RT for $20 \mathrm{~min}$. After rinsing with buffer $(2 \times 5$ min) samples were further incubated with $2 \%$ aqueous osmium tetroxide at RT for 30 min. Following this, samples were washed $(2 \times 5 \mathrm{~min})$ with distilled water and incubated with syringefiltered $1 \%$ aqueous uranyl acetate (UNIVAR) at $4{ }^{\circ} \mathrm{C}$ overnight. Samples were then washed $(3 \times 5$ min) with chilled distilled water and gradually dehydrated with increasing concentrations of ethanol; 10\%, 30\%, 50\%, 70\%, 90\% and 100\% (1 × $7 \mathrm{~min})$ at RT. An Epon 812 resin $20 \mathrm{~mL}$ solution was prepared by initially mixing $12.2 \mathrm{~g}$ of DDSA (Dodecenyl Succinic Anhydride Specially Distilled 13710, Electron Microscopy Sciences), 4.4 g of Araldite (GY 502 10900, Electron Microscopy Sciences) and $6.2 \mathrm{~g}$ of Procure 812 (EMBED 812 RESIN 14900) using a mechanical stirrer. Once the solution was uniformly mixed, $0.8 \mathrm{~mL}$ of BDMA (N-benzyldimethylamine 11400, Electron Microscopy Sciences) was added to it while stirring. Samples were then infiltrated with increasing concentrations of the freshly prepared resin solution in $100 \%$ ethanol at RT and in a sealed container using the following ratios: 1:3 (3 h), 1:2 (3 h), 1:1 (overnight), 2:1 (3 h), 3:1 (3 h). Following this, 
samples were finally infiltrated with $100 \%$ resin solution overnight. Prior to polymerization at $60{ }^{\circ} \mathrm{C}$ for $48 \mathrm{~h}$, the excess resin was drained away by mounting the samples vertically for $1 \mathrm{~h}$.

\section{FIB sectioning and imaging:}

FIB sectioning of SiNW/SiNT substrates with cells was performed using a Thermo Fischer Helios G4 UX FIB-SEM vertically to the sample surface. Prior to FIB sectioning, the region of interest was protected from ion beam (i-beam) damage using i-beam assisted deposition of a $\sim 0.5 \mu \mathrm{m}$ thick platinum layer. The coating was carried out at $30 \mathrm{kV}$ using i-beam current of $0.26-0.44 \mathrm{nA}$, depending on the area size. Following this, rough milling was performed at acceleration voltage of $30 \mathrm{kV}$ and a current of $20 \mathrm{nA}$. The resulting cross sections were then polished with a voltage of $30 \mathrm{kV}$ and a current ranging between $1.2-2.4 \mathrm{nA}$. Images were taken at magnifications of $10 \mathrm{kX}$ and $25 \mathrm{kX}$ using an electron beam at acceleration voltage of $3 \mathrm{kV}$ and a current of $200 \mathrm{pA}$ using immersion mode and with a TLD detector operating in back-scattered (BS) electron collection mode, at a dwell time of 5 $\mu \mathrm{s}$ and $6144 \times 4096$ pixel resolution. During sequential sectioning, images were taken every $20 \mathrm{~nm}$ using previously mentioned e-beam conditions. Original images are black-white inverted.

\section{TEM sample preparation:}

Step 1 - Anti-stick Coating: Samples were inserted into an Oxford Plasma Lab100 ICP-RIE and $\mathrm{C}_{4} \mathrm{~F}_{8}$ deposition was performed with a flow rate of $150 \mathrm{sccm}$ and ICP power of $1000 \mathrm{~W}$. The APC valve position was set at 70 and He pressure was set at 10 Torr. The deposition time was $1 \mathrm{~min}$.

Step 2 - Collagen/Poly-D-lysine(PDL) coating (Optional): $\mathrm{C}_{4} \mathrm{~F}_{8}$-coated samples were immersed in collagen/PDL solution at concentration of $200 \mathrm{ng} / \mu \mathrm{l}$ and placed in a desiccator for $10 \mathrm{~min}$. Following this, samples were left at $4{ }^{\circ} \mathrm{C}$ overnight.

Step 3 - Cell Seeding: GPE86 and HeLa cells were seeded with a concentration of $0.2 \times 10^{6}$ cells $/ \mathrm{ml}$ onto the $\mathrm{C}_{4} \mathrm{~F}_{8}$-coated substrates followed by centrifugation at $250 \mathrm{~g}, 32{ }^{\circ} \mathrm{C}, 15 \mathrm{~min}$. Substrates carrying cells were incubated at $37{ }^{\circ} \mathrm{C}$ with $5 \% \mathrm{CO}_{2}$ for $6 \mathrm{~h}$.

Step 4 - Fixation, Heavy Metal Staining, Dehydration and Resin Embedding: Samples were rinsed with $0.1 \mathrm{M}$ sodium cacodylate buffer and fixed with $2.5 \%$ glutaraldehyde in the same buffer at $4{ }^{\circ} \mathrm{C}$ overnight. Following this, samples were washed $(3 \times 5 \mathrm{~min})$ with chilled $0.1 \mathrm{M}$ sodium cacodylate buffer and quenched with chilled $20 \mathrm{mM}$ glycine solution (Sigma-Aldrich) in the same buffer for 20 min. After repeating the washing step, samples were post-fixed by combining equal volumes of $4 \%$ aqueous osmium tetroxide with $2 \%$ potassium ferrocyanide (UNIVAR) in $0.2 \mathrm{M}$ sodium cacodylate buffer on ice for $1 \mathrm{~h}$. Samples were then washed again $(3 \times 5 \mathrm{~min})$ with chilled buffer and incubated with $1 \%$ tannic acid (BDH) in water at RT for $20 \mathrm{~min}$. After rinsing with buffer $(2 \times 5 \mathrm{~min})$ samples were further incubated with $2 \%$ aqueous osmium tetroxide at RT for $30 \mathrm{~min}$. Following this, samples 
were washed $(2 \times 5 \mathrm{~min})$ with distilled water and incubated with syringe-filtered $1 \%$ aqueous uranyl acetate $(\mathrm{UNIVAR})$ at $4{ }^{\circ} \mathrm{C}$ overnight. Samples were then washed $(3 \times 5 \mathrm{~min})$ with chilled distilled water and gradually dehydrated with increasing concentrations of ethanol; $10 \%, 30 \%, 50 \%, 70 \%$, $90 \%$ and $100 \%(1 \times 7 \mathrm{~min})$ at RT. An Epon 812 resin $20 \mathrm{~mL}$ solution was prepared by initially mixing $12.2 \mathrm{~g}$ of DDSA (Dodecenyl Succinic Anhydride Specially Distilled 13710, Electron Microscopy Sciences), 4.4 g of Araldite (GY 502 10900, Electron Microscopy Sciences) and 6.2 g of Procure 812 (EMBED 812 RESIN 14900) using a mechanical stirrer. Once the solution was uniformly mixed, $0.8 \mathrm{~mL}$ of BDMA (N-benzyldimethylamine 11400, Electron Microscopy Sciences) was added to it while stirring. Samples were then infiltrated with increasing concentrations of the freshly prepared resin solution in 100\% ethanol at RT and in a sealed container using the following ratios: 1:3 (3 h), 1:2 (3 h), 1:1 (overnight), 2:1 (3 h), 3:1 (3 h). Following this, samples were finally infiltrated with $100 \%$ resin solution overnight. The excess resin was drained away by mounting the samples vertically for $1 \mathrm{~h}$. Samples were then placed face down on resin-filled micromolds (ProSciTech) and left for polymerization at $60{ }^{\circ} \mathrm{C}$ for $48 \mathrm{~h}$. After polymerization was completed, substrates were detached using tweezers and resin blocks were released from the micromolds.

Step 5 - Resin Block Trimming and Microtome Sectioning: Each resin block was fixed in a metal specimen holder for ultramicrotomes and inspected under the microscope for the localization of the region of interest (ROI). Following this, resin block was manually trimmed at the sides using a double-edged razor blade so that a pyramid with $45^{\circ}$ angled sides and square surface $(0.5 \mathrm{~mm} \times 0.5$ $\mathrm{mm})$ containing the ROI is formed. Thin $(70 \mathrm{~nm})$ serial sections of the trimmed resin block were then acquired using an ultra-microtome (Leica Ultracut UCT) equipped with a diamond knife. Sections were collected on TEM grids (ProSciTech, formvar/carbon coated, square 100 mesh).

Step 6 - Post-staining of Sections on TEM Grids (Optional): Sections on grids were post-stained with $2 \%$ aqueous uranyl acetate for $20 \mathrm{~min}$ and with lead citrate for $4 \mathrm{~min}$.

\section{TEM imaging:}

TEM was performed using a JEOL 1400Flash operated at $80 \mathrm{kV}$ and images were acquired at magnifications of $300 \mathrm{X}, 800 \mathrm{X}, 2 \mathrm{kX}, 4 \mathrm{kX}, 20 \mathrm{kX}, 25 \mathrm{kX}$ and $30 \mathrm{kX}$.

\section{Ellipsometry measurements:}

$\mathrm{C}_{4} \mathrm{~F}_{8}$ coating thickness measurements were performed using a J. A. Woollam Co., Inc. Variable Angle Spectroscopic Ellipsometer (VASE). 


\section{Endocytosis inhibition study:}

\section{Confocal Imaging:}

Prior to SiNT loading, substrates were treated with ozone for $30 \mathrm{~min}$ to enhance their hydrophilicity. $10 \mu \mathrm{L}$ of Cy5-tagged GFP-encoding mRNA (Cy5-mRNA-GFP, $200 \mu \mathrm{g} / \mathrm{mL}$; Trilink Biotechnology) were loaded onto SiNT substrates and incubated for $1 \mathrm{~h}$ at room temperature (RT). Substrates were then rinsed twice with $1 \times$ DPBS to wash off excessive payloads. GPE86 cells were treated without, and with nystatin $(100 \mu \mathrm{M}$; Sigma-Aldrich) for $1 \mathrm{~h}$ before transferring onto SiNTs pre-loaded with Cy5-mRNA-GFP. After spin $\left(250 \mathrm{~g}, 32{ }^{\circ} \mathrm{C}, 15 \mathrm{~min}\right)$ and $6 \mathrm{~h}$ incubation, cells were stained with Hoechst 33342 for confocal imaging.

Flow Cytometry Analysis: Cells on SiNT substrates were trypsinized with $0.25 \%$ Trypsin-EDTA (Gibco), neutralized with DMEM, transferred to v-bottom 96-well plate, spun down, and washed twice with flow cytometry staining buffer (FACS buffer). Cells were stained with relevant fluorescence markers (Table 1) before proceeding to flow cytometry detection using an LSRIIb flow cytometer $(\mathrm{BD})$.

\section{TEM Imaging:}

GPE86 cells were treated without, and with nystatin (100 $\mu \mathrm{M}$; Sigma-Aldrich) for $1 \mathrm{~h}$ before transferring onto $\mathrm{C}_{4} \mathrm{~F}_{8}$-coated SiNTs. After spin $\left(250 \mathrm{~g}, 32{ }^{\circ} \mathrm{C}, 15 \mathrm{~min}\right)$ and $6 \mathrm{~h}$ incubation, samples were subjected to TEM sample preparation for TEM imaging.

\section{Confocal laser scanning microscopy imaging:}

Fluorescence microscopy images were acquired using a Nikon A1R confocal laser scanning microscope system. Observations were conducted at more than ten different regions on the surface of each sample at magnifications of $20 \mathrm{X}$ and $60 \mathrm{X}$ using a water immersed objective lens. Images were analyzed using the Nikon NIS-Elements Advanced Research software provided by the manufacturer.

Table 1: Fluorescence markers used for confocal microscopy and flow cytometry analysis

\begin{tabular}{|l|l|l|}
\hline Fluorescence markers & $\lambda_{\text {ex }}(\mathbf{n m})$ & $\lambda_{\text {em }}(\mathbf{n m})$ \\
\hline Hoechst 33342 (Hoechst) (Sigma-Aldrich) & 350 & 461 \\
\hline LIVE/DEAD Fixable Aqua stain & 367 & 526 \\
\hline GFP & 488 & 510 \\
\hline Cy5 & 647 & 670 \\
\hline
\end{tabular}




\section{Light microscopy imaging:}

Light microscopy images were acquired using an inverted Nikon Ti-s microscope at two different magnifications: $10 \mathrm{X}$ and $20 \mathrm{X}$.

\section{Data processing and statistical analysis:}

Fluorescence, SEM, and TEM images were processed and analyzed by Image J. Flow cytometry data were analyzed with FlowJo. All statistical analysis was performed using Prism GraphPad 8. Nonparametric two-sided Mann-Whitney U-tests were performed for comparison between two groups.

\section{Safety and hazards:}

! CAUTION Piranha solution is highly corrosive and extremely oxidative. Handling piranha solution in a functioning fume hood without the presence of organic solvent.

! CAUTION Sodium cacodylate irritates the skin and eyes and it is toxic upon inhalation and ingestion. Handle with gloves, goggles, and a mask and work in a fume hood.

! CAUTION Glutaraldehyde is hazardous in the case of contact with the skin or inhalation. Handle with gloves and a mask and work in a fume hood.

! CAUTION Osmium tetroxide is very toxic upon inhalation and ingestion. Avoid contact with the skin and eyes. Handle with gloves, goggles, and a mask and work in a fume hood.

! CAUTION Glycine irritates the skin and eyes and it is toxic upon inhalation and ingestion. Handle with gloves, goggles, and a mask and work in a fume hood.

! CAUTION Potassium ferrocyanide irritates the skin and eyes and it is toxic upon inhalation and ingestion. Handle with gloves, goggles, and a mask and work in a fume hood.

! CAUTION Uranyl acetate is a radioactive material that is fatal if inhaled or swallowed and causes damage to organs after prolonged and repeated exposure. It has a risk of cumulative impact. Handle it with gloves, goggles, and a mask, and work in a fume hood.

! CAUTION Tannic acid is hazardous in the case of contact with the skin and eyes. Avoid inhalation and ingestion. Handle it with gloves and work in a fume hood.

! CAUTION Every reagent involved in Epon 812 resin preparation is hazardous if inhaled or swallowed. Handle with gloves and a mask, and work in a fume hood.

! CAUTION Lead salts are extremely toxic. Handle with gloves, goggles, and a mask and work in a fume hood. 


\section{Additional data}

a.

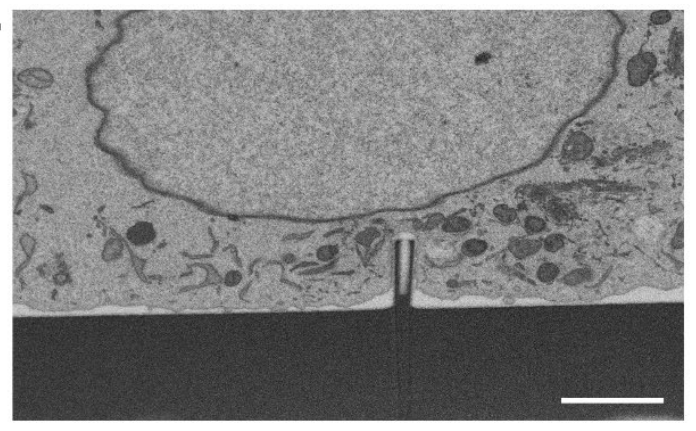

b.

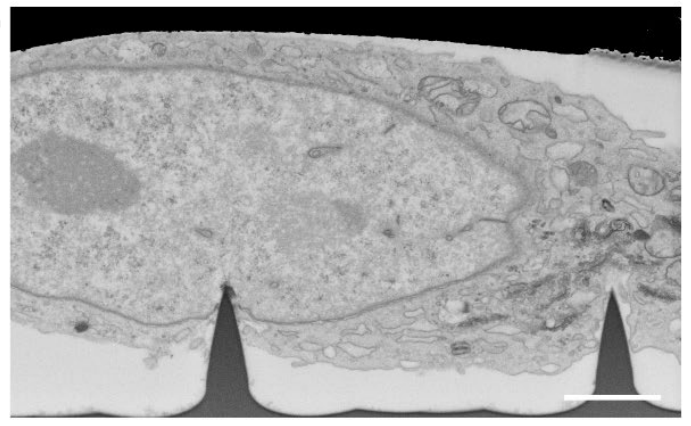

Figure S1: GPE86-SiNT/NW interface. SEM images after FIB milling at $90^{\circ}$ demonstrating the GPE86 membrane deformations induced by (a) SiNTs and (b) SiNWs, respectively. Scale bars: $2 \mu \mathrm{m}$. Original images are black-white inverted.

a.

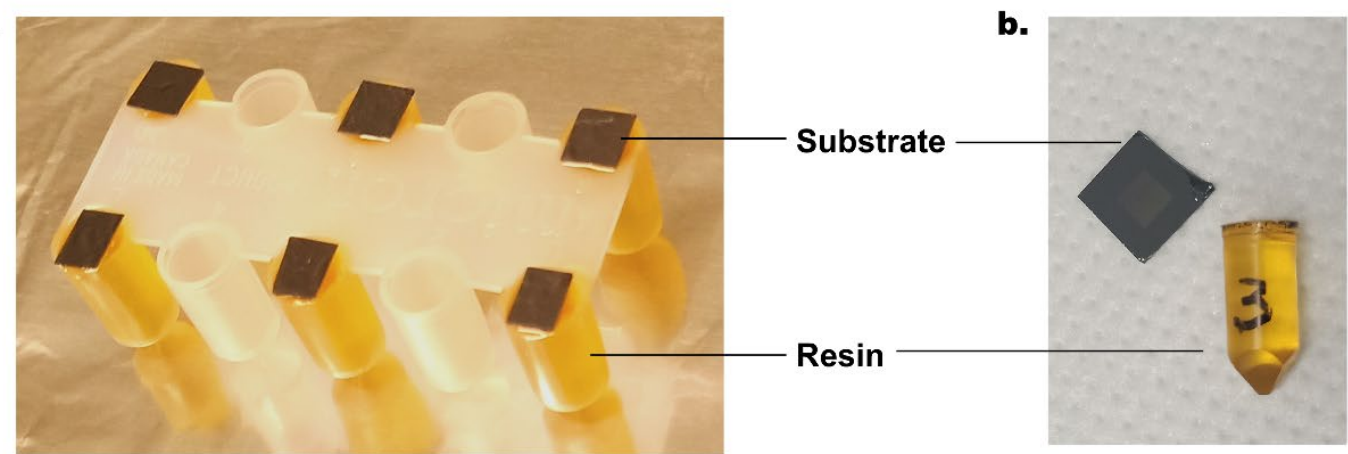

Figure S2: Resin embedding process. a) Resin flat-embedding of cells on SiNT substrates. Substrates are placed face down on resin-filled micromolds and left for polymerization at $60{ }^{\circ} \mathrm{C}$ for $48 \mathrm{~h}$. b) Detachment of a SiNT substrate from its corresponding polymerized resin block. 

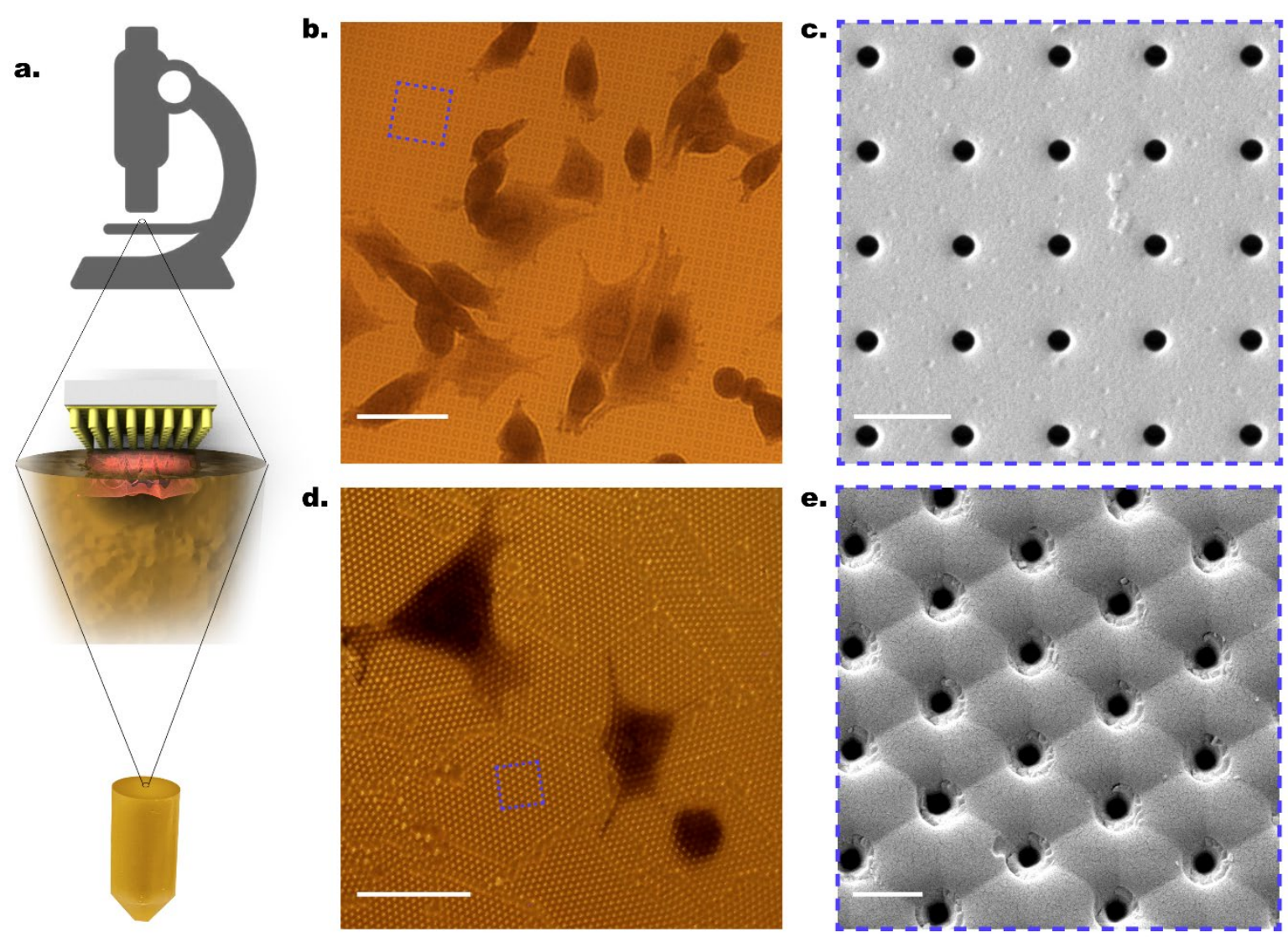

Figure S3: Resin block surface characterization. (a) Light microscopy imaging of the resin block surface showing GPE86 cell resin entrapment, post detachment of (b) a SiNT and (d) a SiNW substrate from the resin block. (c,e) SEM images of the imprint of (c) the SiNT and (e) the SiNW substrate, left in the block. Scale bars: (b,d) $50 \mu \mathrm{m}$, (c) $5 \mu \mathrm{m}$, and (e) $2 \mu \mathrm{m}$. 

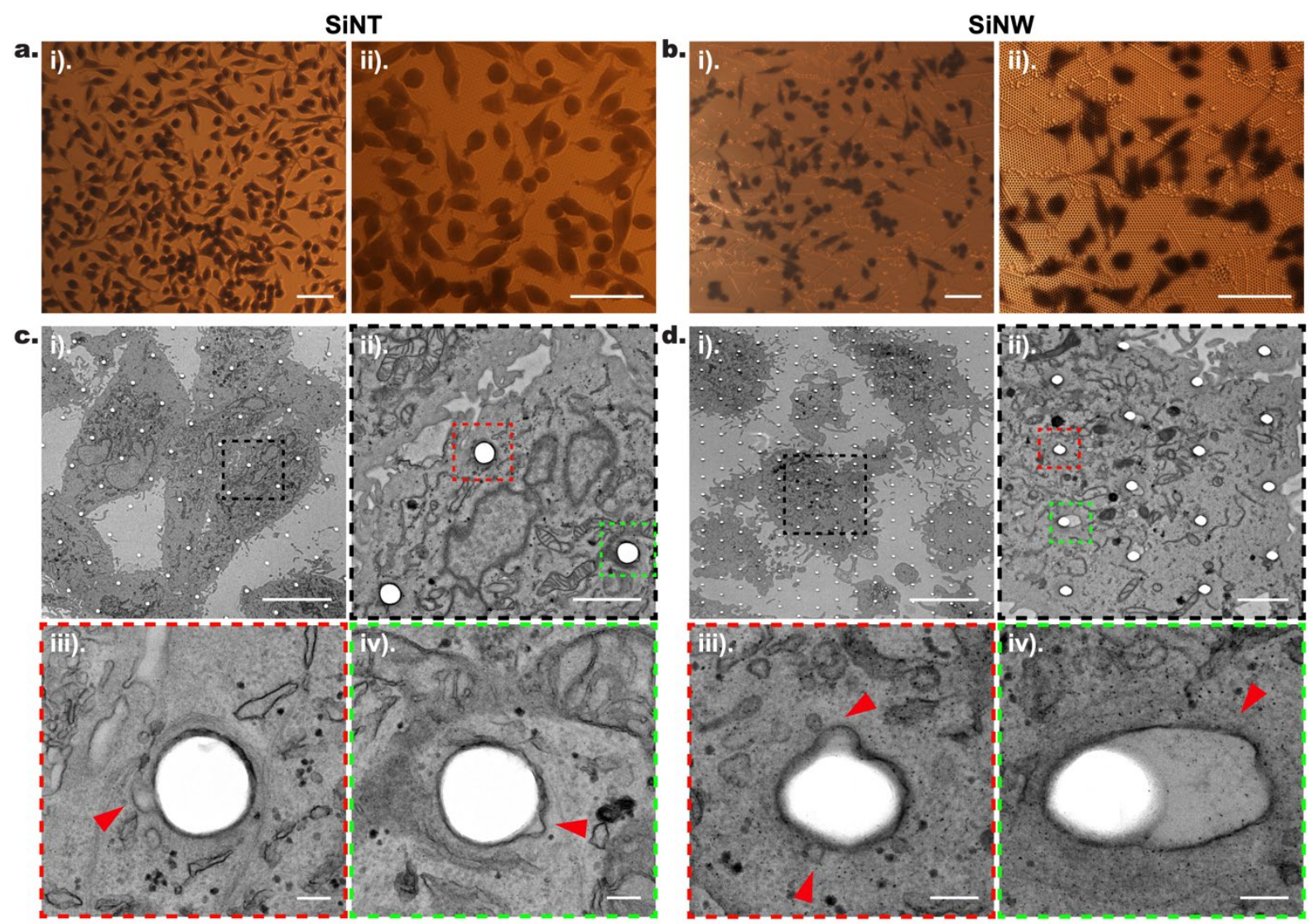

Figure S4: HeLa-SiNT/NW interface. $(a, b)$ Light microscopy images showing (i) zoomout and (ii) zoom-in views of HeLa cell resin entrapment, post detachment of (a) a SiNT substrate and (b) a SiNW substrate from the resin block. Scale bars: $100 \mu \mathrm{m}$. (c,d) TEM images showing (i) low-, (ii) medium-, and (iii, iv) high-magnification views of the interfacial interactions of HeLa cells with the corresponding SiNT and SiNW sites, respectively. Red arrows indicating SiNW/NT-induced membrane curvatures. Scale bars: (i) $10 \mu \mathrm{m}$, (ii) $2 \mu \mathrm{m}$, and (iii,iv) $200 \mathrm{~nm}$. 
a.
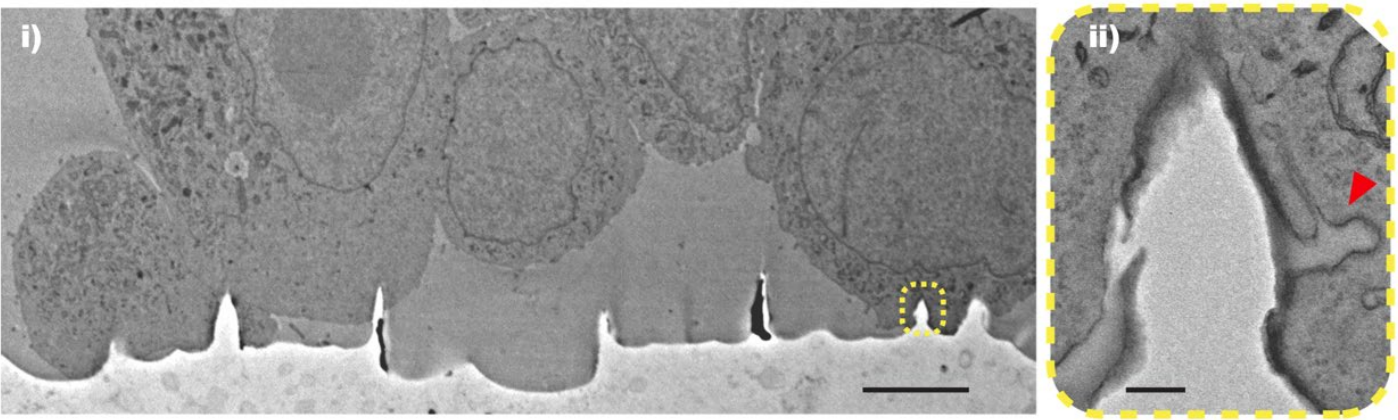

b. 1 i)
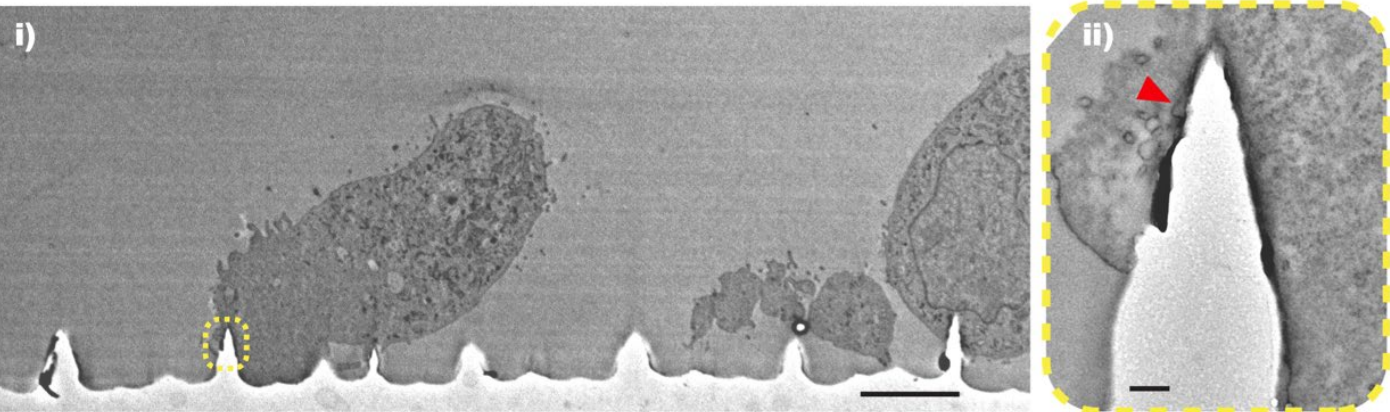

Figure S5: TEM imaging of GPE86-SiNW interface. (a,b) TEM images showing (i) zoom-out and (ii) zoom-in views of vertical cross-sections of GPE86-SiNW interfaces. Red arrows indicating SiNW-induced membrane curvatures. Scale bars: (i) $5 \mu \mathrm{m}$ and (ii) 250 nm.

a.

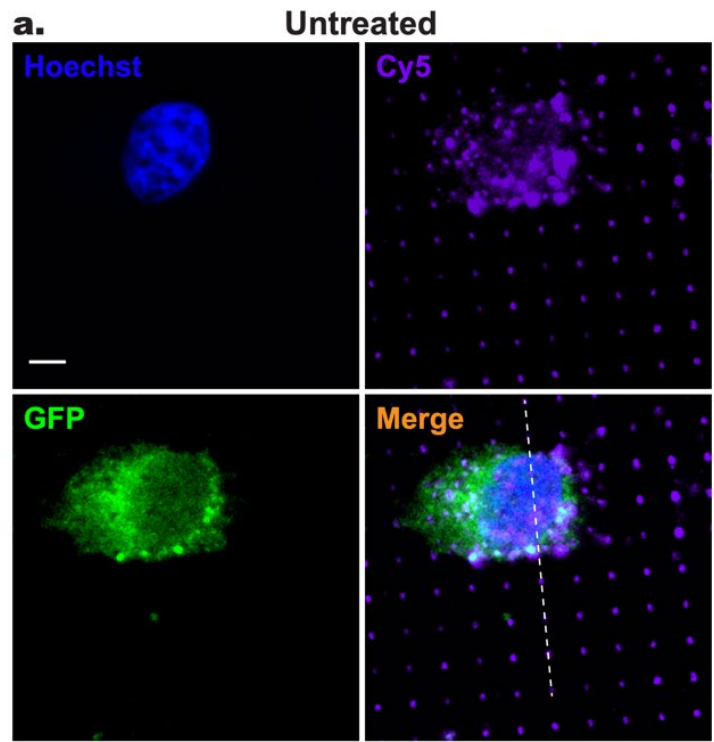

C.

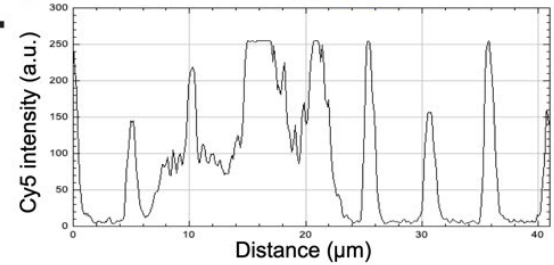

b.

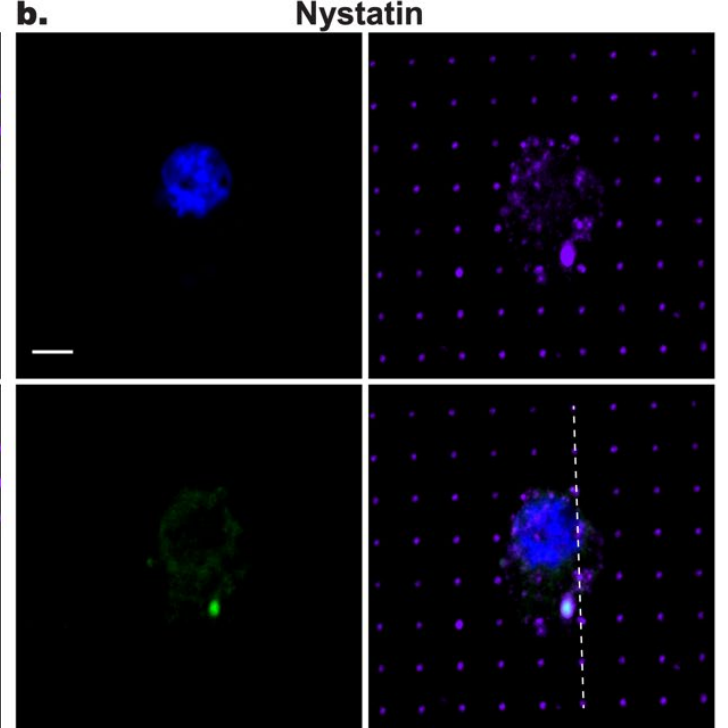

d.

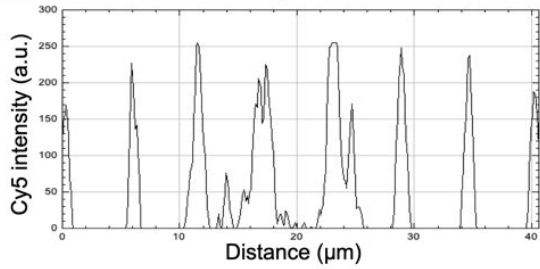

Figure S6: Impact of endocytosis inhibition on SiNT-mediated mRNA delivery. $(a, b)$ Confocal images showing (a) untreated and (b) nystatin-treated GPE86 cells, on SiNTs preloaded with Cy5 (purple)-mRNA-GFP (green); cells were stained with Hoechst 33342 (blue) 
10 min before imaging. Scale bars: $5 \mu \mathrm{m}$. (c,d) Cy5 intensity (arbitrary unit, a.u.) of the corresponding area (dashed line) in the main figures in $(\mathrm{a}, \mathrm{b})$. 